ARTICLE

DOI: $10.1038 / \mathrm{s} 41467-018-05756-7$

\title{
Large anomalous Hall effect in the chiral-lattice antiferromagnet $\mathrm{CoNb}_{3} \mathrm{~S}_{6}$
}

\author{
Nirmal J. Ghimire ${ }^{1}$, A.S. Botana', J.S. Jiang ${ }^{1}$, Junjie Zhang $\mathbb{B}^{1}{ }^{1}$, Y.-S. Chen ${ }^{2}$ \& J.F. Mitchell ${ }^{1}$
}

An ordinary Hall effect in a conductor arises due to the Lorentz force acting on the charge carriers. In ferromagnets, an additional contribution to the Hall effect, the anomalous Hall effect (AHE), appears proportional to the magnetization. While the AHE is not seen in a collinear antiferromagnet, with zero net magnetization, recently it has been shown that an intrinsic AHE can be non-zero in non-collinear antiferromagnets as well as in topological materials hosting Weyl nodes near the Fermi energy. Here we report a large anomalous Hall effect with Hall conductivity of $27 \Omega^{-1} \mathrm{~cm}^{-1}$ in a chiral-lattice antiferromagnet, $\mathrm{CoNb}_{3} \mathrm{~S}_{6}$ consisting of a small intrinsic ferromagnetic component $\left(\approx 0.0013 \mu_{\mathrm{B}}\right.$ per $\left.\mathrm{Co}\right)$ along c-axis. This small moment alone cannot explain the observed size of the AHE. We attribute the AHE to either formation of a complex magnetic texture or the combined effect of the small intrinsic moment on the electronic band structure.

\footnotetext{
${ }^{1}$ Materials Science Division, Argonne National Laboratory, 9700 South Cass Avenue, Argonne, IL 60439, USA. ${ }^{2}$ ChemMatCARS, The University of Chicago, Argonne, IL 60439, USA. Correspondence and requests for materials should be addressed to N.J.G. (email: nghimire@anl.gov)
} 
T he anomalous Hall effect (AHE) is a signature of emergent electromagnetic fields in solids that affect the motion of the electrons, and hence it has been a recent topic of intense study in the context of the topological materials ${ }^{1,2}$. The Hall effect in general is an intrinsic property of a conductor due to the Lorentz force experienced by the charge carriers. In systems with spontaneously broken time-reversal symmetry, an additional contribution, independent of the Lorentz force, is observed, the $\mathrm{AHE}^{1}$. AHE was first observed in ferromagnets where its origin lies in the interplay between spin-orbit coupling (SOC) and magnetization. Reformulation of the SOC-induced intrinsic mechanism of AHE in ferromagnets to the Berry phase curvature in momentum space has been fruitful in predicting and describing the AHE in several other systems, including Weyl (semi)metals ${ }^{3}$, non-collinear antiferromagnets ${ }^{4}$, non-coplanar magnets $^{5-7}$, and other nontrivial spin textures ${ }^{8-11}$. Recent observations of the large anomalous Hall effect in metals with possible Weyl ${ }^{12-14}$ and massive Dirac fermions ${ }^{15,16}$ and/or complex spin textures, e.g., skyrmion bubbles ${ }^{17}$, have generated interest in such materials, especially for the role of correlated topological states in the emergent electronic properties. Here we present a large AHE in $\mathrm{CoNb}_{3} \mathrm{~S}_{6}$ that cannot be understood in terms of conventional mechanisms of the AHE.

$\mathrm{CoNb}_{3} \mathrm{~S}_{6}$ is a member of a large class of intercalated transition metal dichalcogenides, where a $3 d$-transition metal sandwiches between layers of a $5 d$-transition metal dichalcogenide that are coupled by a weak Van der Waals force. $\mathrm{CoNb}_{3} \mathrm{~S}_{6}$ represents the $1 / 3$ fractional intercalation of $\mathrm{Co}$ atoms between the layers of $\mathrm{NbS}_{2}{ }^{18}$. It crystallizes in the hexagonal chiral space group $P 6_{3}$ $22^{19,20}$. It orders antiferromagnetically below $\sim 26 \mathrm{~K}$ and is known to have a rather complex susceptibility for the magnetic field applied parallel to the $c$-axis ${ }^{21,22}$. At $4 \mathrm{~K}$, neutron diffraction measurement has suggested a collinear antiferromagnetic state in which the spins are in the $a b$-plane pointing along a certain crystallographic axis ${ }^{20}$. By itself, however, such a spin structure cannot give rise to the anomalous Hall effect.

The key finding of this work is a large $c$-axis anomalous Hall effect in the antiferromagnetic $\mathrm{CoNb}_{3} \mathrm{~S}_{6}$. Although $\mathrm{CoNb}_{3} \mathrm{~S}_{6}$ shows a small, intrinsic ferromagnetic component $\left(\approx 0.0013 \mu_{\mathrm{B}}\right.$ per Co) along the $c$-axis, this small moment alone cannot explain the observed size of the AHE. Based on its chiral crystal structure and the calculated band structure, we attribute the AHE in $\mathrm{CoNb}_{3} \mathrm{~S}_{6}$ either to the formation of a complex magnetic texture or to an influence of the small intrinsic ferromagnetic moment on the underlying electronic band structure.

\section{Results}

Crystal structure and physical properties of $\mathrm{CoNb}_{3} \mathrm{~S}_{6}$. We verified the room temperature crystal structure of $\mathrm{CoNb}_{3} \mathrm{~S}_{6}$ in the chiral space group $\mathrm{PG}_{3} 22$ by means of single crystal X-ray diffraction (see Supplementary Note 1 and Supplementary Fig. 1). A sketch of the crystal structure adopted by $\mathrm{CoNb}_{3} \mathrm{~S}_{6}$ is shown in Fig. 1a, where the Co atoms occupy the octahedral position between the triangular prismatic layers of the parent compound $2 \mathrm{H}-\mathrm{NbS}_{2}$. Figure $1 \mathrm{~b}$ shows the magnetic susceptibility of $\mathrm{CoNb}_{3} \mathrm{~S}_{6}$ as a function of temperature measured with a magnetic field of $0.1 \mathrm{~T}$ applied along $a$ - and $c$-axis $\left(\chi_{a}\right.$ and $\chi_{c}$, respectively). Over the entire temperature range, $\chi_{c}$ exceeds $\chi_{a}$, consistent with behavior reported in the literature ${ }^{21} \cdot \chi_{a}$ shows a kink at $T_{\mathrm{N}}=$ $27.5 \mathrm{~K}$ corresponding to the antiferromagnetic transition and decreases on further cooling down to $1.8 \mathrm{~K}$. The field cooled (FC) and zero field cooled (ZFC) measurements show identical behavior. Along the $c$-axis, the susceptibility below $T_{\mathrm{N}}$ shows irreversibility between FC and ZFC measurements. The ZFC $\chi_{c}$ shows a behavior similar to that of $\chi_{a}$. However, the FC $\chi_{c}$ increases on cooling below $T_{\mathrm{N}}$, becomes maximum at $25 \mathrm{~K}$ and decreases on further cooling. This increase in FC susceptibility along the $c$-axis near the transition temperature has been reported in previous studies ${ }^{20,21}$ and implies the presence of a small ferromagnetic component along the $c$-axis. With increasing magnetic field, the magnitude of this jump decreases, and at $3 \mathrm{~T}$, FC susceptibility shows a continuous decrease below $T_{\mathrm{N}}$, as shown in the inset of Fig. $1 \mathrm{~b}$, suggesting that the moment along the $c$-axis is suppressed by a sufficiently large magnetic field. At higher temperatures, the susceptibility shows Curie-Weiss behavior. A Curie-Weiss fit to the data between 200 and $300 \mathrm{~K}$ (Supplementary Note 2 and Supplementary Fig. 2) yields the powder averaged effective moment of $3.0 \mu_{\mathrm{B}}$ per Co. This value is smaller than the spin-only moment expected for $\mathrm{Co}^{2+}\left(3.87 \mu_{\mathrm{B}}\right)$ established by neutron diffraction and optical studies ${ }^{20,23}$. Resistivity shows metallic behavior over the measured temperature range of $1.8-300 \mathrm{~K}$, with a sudden drop below $27.5 \mathrm{~K}\left(T_{\mathrm{N}}\right)$, presumably due to the reduction of electron scattering with the onset of the spin ordering (Fig. 1c). $\mathrm{CoNb}_{3} \mathrm{~S}_{6}$ has a very small magnetoresistance of $0.2 \%$ at $2 \mathrm{~K}$ in the field of $9 \mathrm{~T}$ applied along $c$-axis, as shown in the inset of Fig. 1c.

Magnetization. $M$ vs $H$ measured between 29 and $22 \mathrm{~K}$, with the magnetic field along $c$-axis, is shown in Fig. $2 \mathrm{a}$, and the corresponding first-order derivatives are shown in Fig. 2b. Despite the nearly linear $M-H$ curves, a hysteresis with very small remanent magnetization appears below $T_{\mathrm{N}}$, which becomes maximum at 25 $\mathrm{K}\left(0.0013 \mu_{\mathrm{B}}\right.$ per formula unit) and decreases at lower temperatures, as shown in Fig. 2b, c. The hysteresis becomes more clear in $\mathrm{d} M / \mathrm{d} H$ vs $H$ plots shown in Fig. $2 \mathrm{~b}$. At $29 \mathrm{~K}$, which is above $T_{\mathrm{N}}$, $\mathrm{d} M / \mathrm{d} H$ is featureless. At $27 \mathrm{~K}$, when decreasing the field from $6 \mathrm{~T}$ to $-6 \mathrm{~T}$, a peak in $\mathrm{d} M / \mathrm{d} H$ appears at $-1 \mathrm{~T}$. When increasing the field from $-6 \mathrm{~T}$ to $6 \mathrm{~T}$, no peak is seen at $-1 \mathrm{~T}$. Instead, the peak is observed at $1 \mathrm{~T}$. These peaks appear at the coercive field of the hysteresis in $M$ vs $H$. With the decrease in temperature, the coercive field increases and becomes $3 \mathrm{~T}$ at $24 \mathrm{~K}$. No such peaks are seen below $24 \mathrm{~K}$. These data suggest that there is an intrinsic, hard ferromagnetic component along the $c$-axis in the ordered state that is flipped by a temperature-dependent critical field. Switching of this ferromagnetic component gives rise to the hysteresis in $M$ vs $H$. Below $24 \mathrm{~K}$, a magnetic field of $6 \mathrm{~T}$ cannot switch the ferromagnetic component. As a result, no hysteresis is observed. Application of a larger magnetic field is expected to reveal the hysteresis even below $24 \mathrm{~K}$. In fact, this hysteresis is evident in the Hall effect measurement presented below. In Fig. 2c we show the ferromagnetic component as a function of the magnetic field obtained by subtracting a linear antiferromagnetic background from the $M$ vs $H$ curves presented in Fig. 2a. On the other hand, when magnetic field is applied along the $a$-axis, $M$ vs $H$ shows linear behavior at all temperatures measured, as depicted in Fig. 2d-f.

Hall effect. Hall resistivity $\left(\rho_{y x}\right)$ vs $H$ measured between 28 and $23 \mathrm{~K}$ with current along $a$-axis and magnetic field along $c$-axis is depicted in Fig. 3a. The Hall effect at each temperature was measured by cooling the sample in a magnetic field of $9 \mathrm{~T}$ and then by changing the magnetic field from 9 to $-9 \mathrm{~T}$ and subsequently from -9 to $9 \mathrm{~T}$. At $28 \mathrm{~K}$, which is just above $T_{\mathrm{N}}$, the Hall resistivity is linear, as expected for a normal conductor. The sign of the Hall resistivity indicates that holes are the majority charge carriers in $\mathrm{CoNb}_{3} \mathrm{~S}_{6}$. Within the single band model, the estimated carrier concentration $\left(n=1 /\left|e R_{0}\right|\right)$ is $2.49 \times 10^{21} \mathrm{~cm}^{-3}$, where $e$ is the charge of an electron and $R_{0}$ is the ordinary Hall coefficient. When the temperature is lowered below $T_{\mathrm{N}}$, an additional contribution appears in the Hall resistivity. At $27 \mathrm{~K}$, this anomalous 


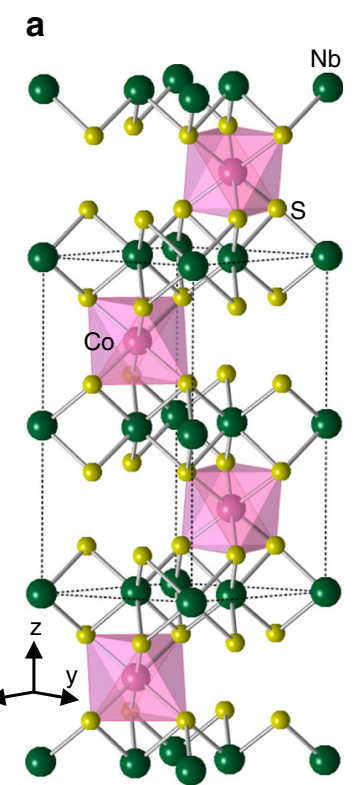

b
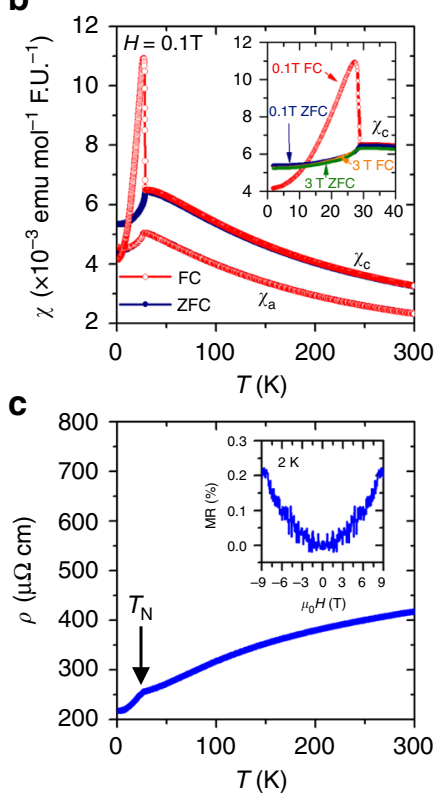

Fig. 1 Crystal structure and characteristics of $\mathrm{CoNb}_{3} \mathrm{~S}_{6}$. a Sketch of crystal structure of $\mathrm{CoNb}_{3} \mathrm{~S}_{6}$. Each $\mathrm{Co}$ atom is octahedrally coordinated with $\mathrm{S}$ atoms. $\mathbf{b}$ Magnetic susceptibility measured with magnetic field along $a$ - and c-axis. c Electrical resistivity as a function of temperature measured with current along $a$-axis. Inset shows the magnetoresistance defined by $\mathrm{MR}=$ $\left(\rho_{H}-\rho_{0}\right) / \rho_{0} \times 100 \%$, where $\rho_{H}$ is the resistivity measured at the magnetic field $H$ and $\rho_{0}$ the resistivity measured at $H=0$. The MR was measured with current along $a$-axis and magnetic field along $c$-axis

Hall signal is small. It increases both in magnitude and in field with decreasing temperature, becoming maximum at $23 \mathrm{~K}$. Below $23 \mathrm{~K}$, no switching behavior of the Hall resistivity is observed when measured with the maximum field of $9 \mathrm{~T}$. However, the Hall resistivity increases down to $20 \mathrm{~K}$ (Supplementary Note 3 and Supplementary Fig. 3). At $15 \mathrm{~K}$, a very small AHE is observed, and at $2 \mathrm{~K}$ there is no anomalous Hall effect. These observations reveal two important points. (1) Hysteresis in the AHE is observed when the magnetic field switches the small FM component (Fig. 2a-c). (2) The AHE is observed due to the stabilization of the FM component, which is observed between $T_{\mathrm{N}}$ and $23 \mathrm{~K}$. At $20 \mathrm{~K}$, a magnetic field of $9 \mathrm{~T}$ cannot switch the FM component, and hence the AHE does not switch sign, but gives the same large value. At $2 \mathrm{~K}$, a magnetic field of $9 \mathrm{~T}$ cannot induce the FM component at all. As a result, there is no AHE observed for $|H| \leq 9 \mathrm{~T}$.

Conventionally, the Hall resistivity in a ferromagnet is given by the relation $\rho_{y x}=R_{0} B+\rho_{\mathrm{yx}}^{\mathrm{A}}$, where $R_{0}$ is the ordinary Hall coefficient, $B=\mu_{0} H$, and $\rho_{\mathrm{yx}}^{\mathrm{A}}$ is the anomalous Hall resistivity ${ }^{1}$. In Fig. 3a, we see that the Hall resistivity measured in the paramagnetic state, i.e., at $28 \mathrm{~K}$ and in the antiferromagnetic state, except near the switching fields has the same slope. This indicates that $R_{0} B$ is constant between 28 and $23 \mathrm{~K}$, and allows us to subtract the ordinary Hall resistivity to obtain the anomalous part. Figure $3 \mathrm{~b}$ shows $\rho_{\mathrm{yx}}^{\mathrm{A}}$ vs $H$, where $\rho_{\mathrm{yx}}^{\mathrm{A}}$ is obtained by subtracting the data measured at $28 \mathrm{~K}$ from $\rho_{y x}$ measured below $T_{\mathrm{N}}$. As there is a finite ferromagnetic component along the $c$-axis, we first examine the effect of this small ferromagnetic component on the anomalous Hall effect. For a ferromagnet, $\rho_{\mathrm{yx}}^{\mathrm{A}}=R_{\mathrm{S}} \mu_{0} M$, where $R_{\mathrm{S}}$ is the anomalous Hall coefficient, $\mu_{0}$ is the permeability, and $M$ is the magnetization. $R_{\mathrm{S}}$ scales the $M-H$ curve to the anomalous part of the Hall resistivity. To check for this scaling in $\mathrm{CoNb}_{3} \mathrm{~S}_{6}$, we obtained the magnetization of the ferromagnetic component by subtracting a straight line from the $M$ vs $H$ curve measured at a representative temperature of $25 \mathrm{~K}$, which shows the maximum remanent magnetization. The obtained magnetization multiplied by $-1,(-\Delta M)$ vs $H$ is plotted in Fig. 3c. The anomalous Hall resistivity measured at $25 \mathrm{~K}$ is also plotted in the same figure. These plots show that the anomalous Hall effect does scale with magnetization. $R_{\mathrm{S}}$ is negative and has an exceedingly large value compared to $R_{0}$. $R_{\mathrm{S}}$ estimated from this scaling at $25 \mathrm{~K}$ is $-1.41 \times 10^{-4} \mathrm{~m}^{3} \mathrm{C}^{-1}$, which is four orders of magnitude larger than $R_{0}=2.4 \times 10^{-9} \mathrm{~m}^{3} \mathrm{C}^{-1}$. In a canonical ferromagnet-like $\mathrm{Fe}^{24}$ or ferromagnets with closely related and even the same crystal structure as $\mathrm{CoNb}_{3} \mathrm{~S}_{6}$, such as $\mathrm{Fe}_{1 / 4} \quad \mathrm{TaS}_{2}{ }^{25}$ and $\mathrm{Fe}_{1 / 3} \mathrm{TaS}_{2}{ }^{26}, R_{\mathrm{S}} / \mathrm{R}_{0} \approx 100$, two orders of magnitude smaller than that in $\mathrm{CoNb}_{3} \mathrm{~S}_{6}$. This significant boost of the coefficient of AHE in $\mathrm{CoNb}_{3} \mathrm{~S}_{6}$ is unlikely to be accounted for by the effect of the weak $c$-axis ferromagnetic component alone and therefore must have an additional contribution.

The contribution of the ferromagnetic component to the anomalous Hall resistivity, i.e., $R_{\mathrm{S}}$ can be estimated from the high magnetic field part of the anomalous Hall resistivity vs magnetization plot. As $\rho_{\mathrm{yx}}^{\mathrm{A}}=R_{\mathrm{S}} \mu_{0} M$, the slope of $\rho_{\mathrm{yx}}^{\mathrm{A}}$ vs $M$ gives $\mu_{0} R_{\mathrm{S}}$. In Fig. $3 \mathrm{~d}$, we see that $\rho_{\mathrm{yx}}^{\mathrm{A}}$ vs $M$ resembles $\rho_{\mathrm{yx}}^{\mathrm{A}}$ vs $H$ such that $\rho_{\mathrm{yx}}^{\mathrm{A}}$ changes sign at the coercive fields. After the abrupt sign change, $\rho_{\mathrm{yx}}^{\mathrm{A}}$ shows a linear variation with $M$. This linear behavior of $\rho_{\mathrm{yx}}^{\mathrm{A}}$ vs $M$ at high field arises from the continuous spin tilting by the magnetic field toward the $c$-axis and represents the contribution from the field-induced ferromagnetic component. The slope in this linear regime gives $\mu_{0} R_{\mathrm{S}}$ for this compound, which is represented by dashed lines in Fig. $3 \mathrm{~d}$. $R_{\mathrm{S}}$ estimated from this slope is $-1.12 \times 10^{-7} \mathrm{~m}^{3} \mathrm{C}^{-1}$, which is comparable to that of the ferromagnet $\mathrm{Fe}_{1 / 3} \mathrm{TaS}_{2}{ }^{26}$. The extra component in the anomalous Hall resistivity, which we label as $\rho_{\mathrm{yx}}^{\mathrm{A}^{\prime}}$ can be obtained by subtracting this linear part from the anomalous Hall effect: $\rho_{\mathrm{yx}}^{\mathrm{A}^{\prime}}=\rho_{\mathrm{yx}}^{\mathrm{A}}-R_{\mathrm{S}} \mu_{0} M$ and is shown in Fig. 3e, which shows that contribution from the FM component from spin tilting is negligibly small compared to $\rho_{\mathrm{yx}}^{\mathrm{A}}$. In Fig. $3 \mathrm{f}$, we plot the anomalous Hall conductivity $\sigma_{\mathrm{xy}}^{\mathrm{A}}=\rho_{\mathrm{yx}}^{\mathrm{A}} /\left[\left(\rho_{\mathrm{yx}}^{\mathrm{A}}\right)^{2}+\left(\rho_{\mathrm{xx}}\right)^{2}\right]$ as a function of magnetic field between 27 and $23 \mathrm{~K}$. The anomalous Hall conductivity at $23 \mathrm{~K}$ takes the value $27 \Omega^{-1} \mathrm{~cm}^{-1}$. A similar behavior, in which a very small ferromagnetic component and its anomalously large scaling with the anomalous Hall resistivity, has been observed in $\mathrm{Mn}_{3} \mathrm{Sn}$ and $\mathrm{Mn}_{3} \mathrm{Ge}$. Here, the anomalous Hall effect has been attributed to the combined effect of non-collinear antiferromagnetic spin texture and electronic band structure ${ }^{12,13}$. A large anomalous Hall coefficient, reported for $\mathrm{Fe}_{3} \mathrm{Sn}_{2}{ }^{15,16}$ cannot be described by the ferromagnetic component alone and is believed to be due to the massive quasi-2D Dirac cones near the Fermi energy. Likewise, the large AHE observed in frustrated systems such as $\operatorname{Pr}_{2} \mathrm{Ir}_{2} \mathrm{O}_{7}$ has been attributed to the non-coplanar spin texture ${ }^{27}$.

\section{Discussion}

We now discuss possible origins of the anomalous Hall effect in $\mathrm{CoNb}_{3} \mathrm{~S}_{6}$. First, we can rule out impurity-related extrinsic mechanisms of the AHE from the fact that the AHE vanishes at low temperatures, where the laboratory magnetic fields are insufficient to alter spins state, e.g., at $2 \mathrm{~K}$ as shown in Fig. $3 \mathrm{a}$ and Supplementary Fig. 3a. Second, a collinear antiferromagnetic state cannot give rise to the anomalous Hall effect. Finally, we showed above that an FM component due to simple magnetic fieldinduced spin tilt alone cannot account for the observed AHE. We thus consider other scenarios for the origin of the AHE in 


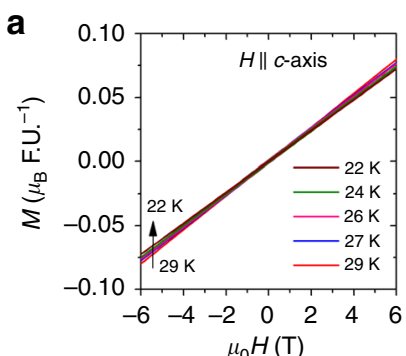

b
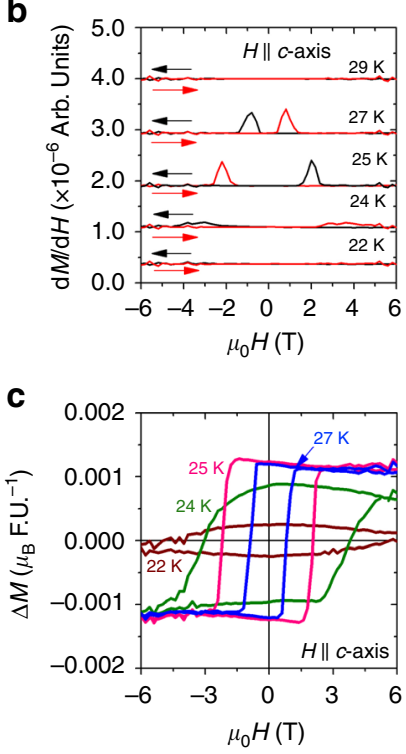

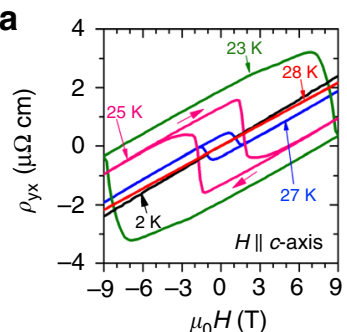

b

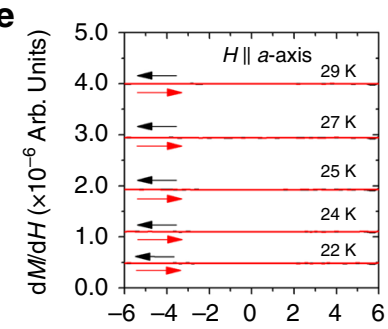

$\mu_{0} H(\mathrm{~T})$
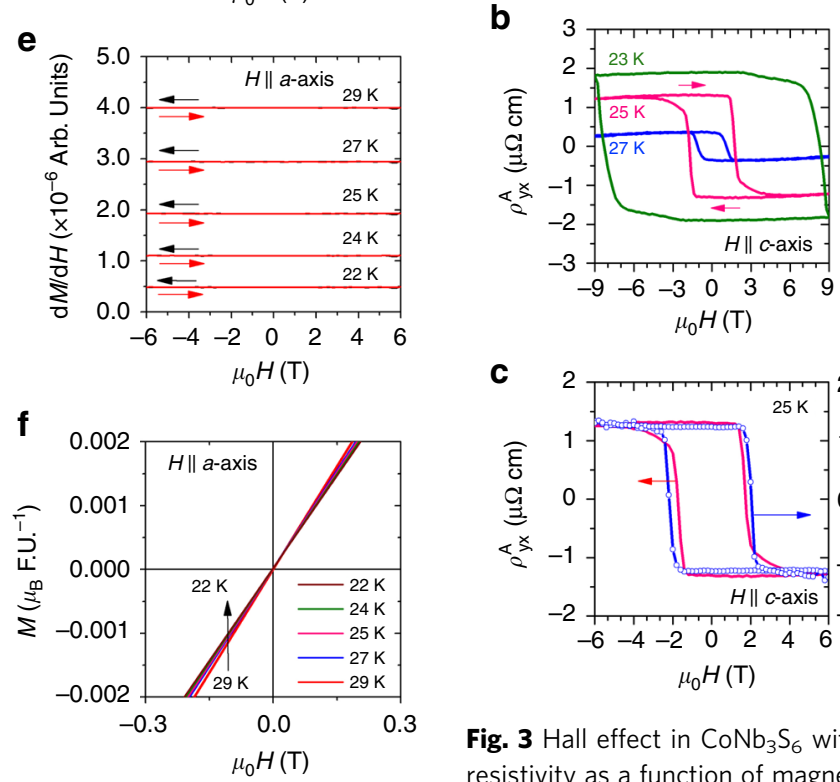

Fig. 2 Magnetization of $\mathrm{CoNb}_{3} \mathrm{~S}_{6}$. a $\mathrm{M}$ vs $\mathrm{H}$ measured with magnetic field applied along the $c$-axis. $\mathbf{b} \mathrm{d} M / \mathrm{d} H$ vs $H$ for magnetic field applied along the $c$-axis. The black (red) curves represent $\mathrm{d} M / \mathrm{d} H$ for decreasing (increasing) $H$. c Ferromagnetic moment $(\Delta M)$ along the $c$-axis obtained by subtracting the antiferromagnetic background from the measured $M$ vs $H$ presented in a. d $M$ vs $H$ for magnetic field applied along the $a$-axis. e $\mathrm{d} M / \mathrm{d} H$ vs $H$ for magnetic field applied along the $a$-axis. The black (red) curves represent $\mathrm{d} M / \mathrm{d} H$ for decreasing (increasing) $H$. $\mathbf{f}$ Low field $M$ vs $H$ measured with magnetic field along $a$-axis. $\mathrm{d} M / \mathrm{d} H$ plots in $\mathbf{b}$ and $\mathbf{e}$ are shifted by a constant factor for clarity

$\mathrm{CoNb}_{3} \mathrm{~S}_{6}$ : (1) non-collinear, non-coplanar or other complex magnetic textures, or (2) interplay of electronic and magnetic degrees of freedom.

$\mathrm{CoNb}_{3} \mathrm{~S}_{6}$ has a chiral crystal structure. In a magnet with such a chiral lattice, competition among the exchange interaction, Dzyaloshinskii-Moriya interaction (DMI), magnetocrystalline anisotropy, and Zeeman energy may result in complex magnetic textures ${ }^{28-30}$, including non-collinear or non-coplanar spins that can give rise to large $\mathrm{AHE}^{10-13,27,31}$. The neutron diffraction experiment reported by Parkin et al. ${ }^{20}$ revealed no such complex spin structure. However, this is not unexpected given (1) the measurement is carried out in zero field at $4 \mathrm{~K}$, where our data does not show AHE when measured up to the magnetic field of 9 $\mathrm{T}$, and (2) a diffraction experiment such as that carried out by Parkin et al. would be insensitive to the large-scale spin structures. Thus, the nature of the spin structure in $\mathrm{CoNb}_{3} \mathrm{~S}_{6}$ near $T_{\mathrm{N}}$ remains an open question.

Figure $4 \mathrm{a}-\mathrm{c}$ shows the paramagnetic band structure of $\mathrm{CoNb}_{3} \mathrm{~S}_{6}$ without inclusion of SOC. The metallic character of $\mathrm{CoNb}_{3} \mathrm{~S}_{6}$ is evident by the presence of the hole pockets along $\Gamma$ $-A$, which is consistent with the measured hole-like character of the charge carriers. There are two linear electron and hole band crossings along $\Gamma-M$ and $K-\Gamma$. Inclusion of SOC (Fig. 4b) opens
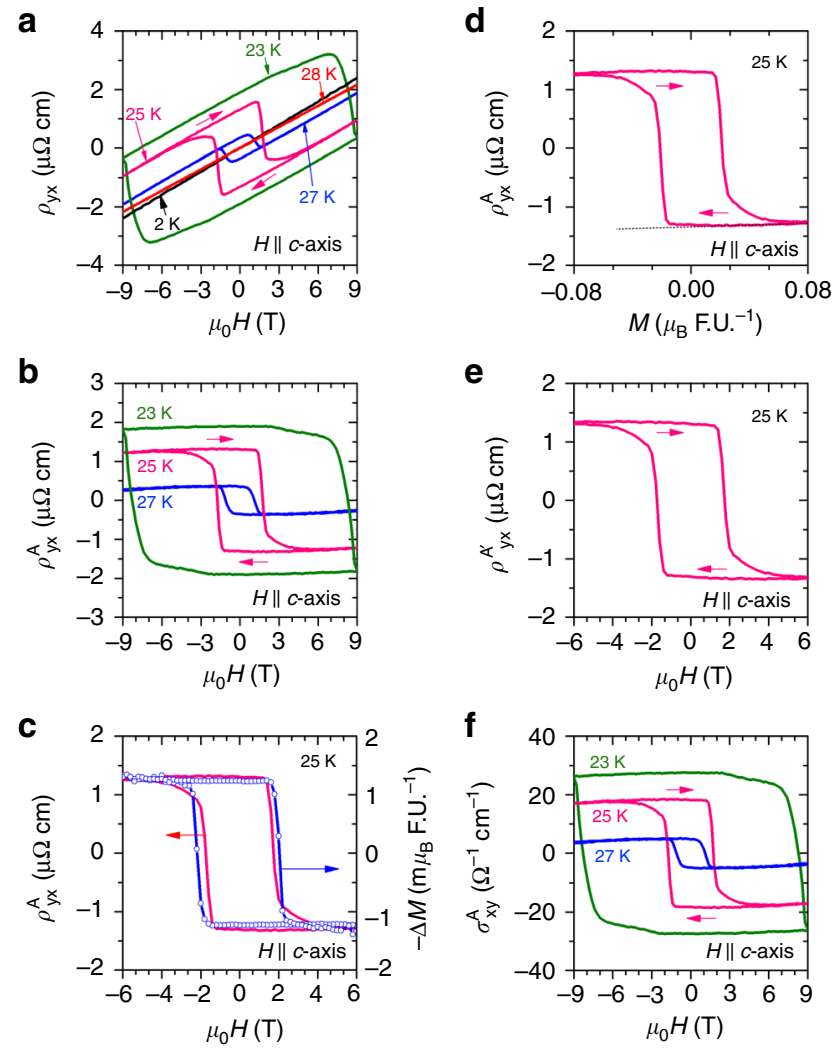

Fig. 3 Hall effect in $\mathrm{CoNb}_{3} \mathrm{~S}_{6}$ with magnetic field along c-axis. a Hall resistivity as a function of magnetic field. $\mathbf{b}$ Anomalous Hall resistivity as a function of magnetic field obtained by subtracting the ordinary Hall resistivity $\rho_{y x}$ as discussed in the text. c Scaling of the Hall resistivity and the ferromagnetic moment. d Anomalous Hall resistivity as a function of magnetization. The straight line represents the slope of the high field $\rho_{\mathrm{yx}}^{\mathrm{A}} \mathrm{vs}$ $M$ that gives the anomalous Hall part due to the ferromagnetic component from the field-induced spin tilt. e Anomalous Hall resistivity obtained by subtracting the estimated Hall contribution form the ferromagnetic component of magnetic field-induced spin tilt as a function of magnetic field. $\mathbf{f}$ Hall conductivity as a function of magnetic field

a small gap at the band crossings along these lines and splits the bands due to the lack of inversion symmetry ${ }^{32,33}$. The band structure along $\Gamma-A$ is enlightening in this regard. In the calculation with SOC (Fig. $4 \mathrm{a}$ ) both at $\Gamma$ and $A$, two doubly degenerate bands touch at a point $\approx 40 \mathrm{meV}$ above the Fermi energy $\left(E_{\mathrm{F}}\right)$. The splitting of these bands due to SOC along $\Gamma-A$ (Fig. 4b) is remarkably large and results in the formation of several linearly crossing bands and avoided crossings. In Fig. 4d, we show the band dispersion along $A-\Gamma-A$ near $E_{\mathrm{F}}$ (see Supplementary Note 4 and Supplementary Fig. 4 for symmetry analysis). Bands cross linearly at all of these points. Two of these points lie within $\approx 15$ $\mathrm{meV}$ of $E_{\mathrm{F}}$. It has been established rigorously that in nonmagnetic crystals and in directions parallel to a $6_{3}$ screw axis (along $\Gamma-A$ in $\mathrm{CoNb}_{3} \mathrm{~S}_{6}$ ), band degeneracies at the $\Gamma$ points are Weyl nodes ${ }^{34}$. The $\mathrm{AHE}$ in $\mathrm{CoNb}_{3} \mathrm{~S}_{6}$ is observed only when the external magnetic field induces a small moment along $c$-axis, which suggests that the large enhancement in AHE may result due to the combined effect of such a field in the presence of the near $E_{\mathrm{F}}$ Weyl nodes.

In summary, $\mathrm{CoNb}_{3} \mathrm{~S}_{6}$ is a member of the class of antiferromagnets that show a large anomalous Hall effect. The large AHE is not explained by the reported collinear antiferromagnetic structure, and we suggest the formation of complex, noncollinear magnetic textures or an interplay between the magnetic texture 

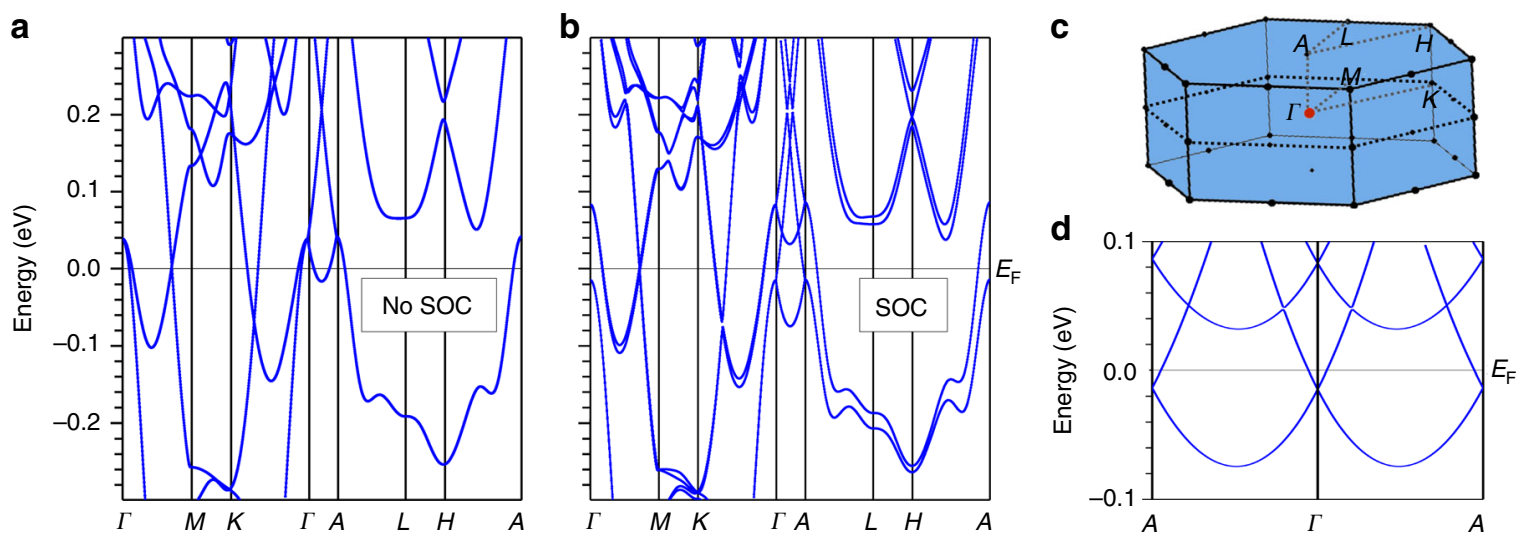

Fig. 4 Electronic band structure of $\mathrm{CoNb}_{3} \mathrm{~S}_{6}$. a Without inclusion of spin-orbit coupling and $\mathbf{b}$ with inclusion of spin-orbit coupling. Spin-orbit coupling causes splitting of the bands, which is most pronounced along $\Gamma-A$. c Brillouin zone of $\mathrm{CoNb}_{3} \mathrm{~S}_{6}$. d Electronic band structure (with $\mathrm{SOC}$ ) near the Fermi energy along the $A-\Gamma-A$ high symmetry line

and electronic band structure as two possible mechanisms for the large AHE. $\mathrm{CoNb}_{3} \mathrm{~S}_{6}$ is a member of a large family of the $1 / 3$ intercalated $\mathrm{TX}_{2}$ compounds, where $\mathrm{T}$ is a 4 - or 5 - $d$ transition metal element, and $\mathrm{X}$ is a chalcogen $(\mathrm{S}, \mathrm{Se})$. As the change in the intercalated $3 d$ element changes the nature of both magnetic and electronic structure, our realization of the AHE in $\mathrm{CoNb}_{3} \mathrm{~S}_{6}$ opens a platform to explore and perhaps manipulate the interplay among spin texture, electronic band structure, and the associated emergent phenomena in a large class of poorly explored materials.

\section{Methods}

Crystal growth and characterization. Single crystals of $\mathrm{CoNb}_{3} \mathrm{~S}_{6}$ were grown by chemical vapor transport using iodine as the transport agent. First, a polycrystalline sample was prepared by heating stoichiometric amounts of cobalt powder (Alfa Aesar 99.998\%), niobium powder (Johnson Matthey Electronics 99.8\%), and sulfur pieces (Alfa Aesar 99.9995\%) in an evacuated silica ampoule at $900^{\circ} \mathrm{C}$ for 5 days. Subsequently, $2 \mathrm{~g}$ of the powder was loaded together with $0.5 \mathrm{~g}$ of iodine in a fused silica tube of $14 \mathrm{~mm}$ inner diameter. The tube was evacuated and sealed under vacuum. The ampoule of $11 \mathrm{~cm}$ length was loaded in a horizontal tube furnace in which the temperature of the hot zone was kept at $950^{\circ} \mathrm{C}$ and that of the cold zone was $\approx 850^{\circ} \mathrm{C}$ for 7 days. Several $\mathrm{CoNb}_{3} \mathrm{~S}_{6}$ crystals formed with a distinct, wellfaceted flat plate-like morphology. The crystals of $\mathrm{CoNb}_{3} \mathrm{~S}_{6}$ were examined by single crystal X-ray diffraction at beamline 15-ID-D at the APS, Argonne National Laboratory (ANL), where the data were collected with an APEX2 Area Detector using synchrotron radiation $(\lambda=0.41328 \AA)$ at $293 \mathrm{~K}$. Compositional analysis was done using an energy dispersive X-ray spectroscopy (EDS) at the Electron Microscopy Center, ANL.

Magnetic and transport property measurements. Magnetization measurements were made using a Quantum Design VSM SQUID. Both in FC and ZFC mode, susceptibility data were measured by sweeping temperature up from $1.8 \mathrm{~K}$. In the derivative of $M$ vs $H$, we observed a small peak around $H=0$ both above and below $T_{\mathrm{N}}$ and both along $a$ - and $c$-axis, possibly due to an unknown paramagnetic impurity. For data at all temperatures presented in Fig. 2, a background measured at $30 \mathrm{~K}$ was subtracted. A small asymmetry of the peak position in the $\mathrm{d} M / \mathrm{d} H$ vs $H$ was observed when only one loop of $M$ vs $H$ was measured. We did not see such an asymmetry when a second loop of the $M$ vs $H$ was measured, which is presented in Fig. 2. Transport measurements were performed on a quantum design PPMS following a conventional 4-probe method. Au wires of $25 \mu \mathrm{m}$ diameter were attached to the sample with Epotek H20E silver epoxy. An electric current of $1 \mathrm{~mA}$ was used for the transport measurements. In magnetoresistance measurements, the contact misalignment was corrected by field symmetrizing the measured data. The following method was adopted for the contact misalignment correction in Hall effect measurements. The Hall resistance was measured at $H=0$ by decreasing the field from the positive magnetic field $\left(R_{H_{+}}\right)$, where $H$ represents the external magnetic field. Again the Hall resistance was measured at $H=0$ by increasing the field from negative magnetic field $\left(R_{H_{-}}\right)$. Average of the absolute value of $\left(R_{H_{+}}\right)$ and $\left(R_{H_{-}}\right)$was then subtracted from the measured Hall resistance. The conventional antisymmetrization method was also used for the Hall resistance measured at $28 \mathrm{~K}$ (above $T_{\mathrm{N}}$ ) and at $2 \mathrm{~K}$ (where no anomalous Hall effect was observed), which gave same result as obtained from the former method.
Electronic structure calculations. The electronic structure calculations were carried out within density functional theory (DFT) using the all-electron, full potential code WIEN $2 \mathrm{~K}^{35}$ based on the augmented plane wave plus local orbital $(\mathrm{APW}+\mathrm{lo})$ basis set ${ }^{36}$. The Perdew-Burke-Ernzerhof (PBE) version of the generalized gradient approximation (GGA) ${ }^{37}$ was chosen as the exchange correlation potential. Spin-orbit coupling (SOC) was introduced in a second variational procedure $^{38}$. A dense $k$-mesh of $28 \times 28 \times 12$ was used for the Brillouin zone (BZ) sampling. A $R_{\mathrm{MT}} K_{\max }$ of 7.0 was chosen for all calculations. Muffin tin radii were 2.5 a.u. for $\mathrm{Nb}, 2.45$ a.u. for $\mathrm{Co}$, and 2.01 a.u. for $\mathrm{S}$.

Data availability. The authors declare that the main data supporting the findings of this study are available within the article and its Supplementary Information files. Extra data are available from the corresponding author on request.

Received: 12 March 2018 Accepted: 2 July 2018

Published online: 16 August 2018

\section{References}

1. Nagaosa, N., Sinova, J., Onoda, S., McDonald, A. H. \& Ong, N. P. Anomalous Hall effect. Rev. Mod. Phys. 82, 1539-1592 (2010).

2. Nagaosa, N., Yu, X. Z. \& Tokura, Y. Gauge fields in real and momentum spaces in magnets: monopoles and skyrmions. Philos. Trans. R. Soc. A 370, 5806-5819 (2012).

3. Burkov, A. A. Anomalous Hall effect in Weyl metals. Phys. Rev. Lett. 113, $187202(2014)$

4. Chen, H., Niu, Q. \& MacDonald, A. H. Anomalous Hall effect arising from noncollinear antiferromagnetism. Phys. Rev. Lett. 112, 017205 (2014).

5. Shindou, R. \& Nagaosa, N. Orbital ferromagnetism and anomalous Hall effect in antiferromagnets on the distorted fcc lattice. Phys. Rev. Lett. 87, 116801 (2001).

6. Ohgushi, K., Murakami, S. \& Nagaosa, N. Spin anisotropy and quantum Hall effect in the kagome lattice: chiral spin state based on a ferromagnet. Phys. Rev. B 62, R6065 (2000)

7. Martin, I. \& Batista, C. D. Itinerant electron-driven chiral magnetic ordering and spontaneous quantum Hall effect in triangular lattice models. Phys. Rev. Lett. 101, 156402 (2008).

8. Schulz, T. et al. Emergent electrodynamics of skyrmions in a chiral magnet. Nat. Phys. 8, 301-304 (2012).

9. Nagaosa, N. \& Tokura, Y. Topological properties and dynamics of magnetic skyrmions. Nat. Nanotechnol. 8, 899-911 (2013).

10. Neubauer, A. et al. Topological Hall effect in the A phase of MnSi. Phys. Rev. Lett. 102, 186602 (2009).

11. Kanazawa, N. et al. Large topological Hall effect in a short-period helimagnet MnGe. Phys. Rev. Lett. 106, 156603 (2011).

12. Nakatsuji, S., Kiyohara, N. \& Higo, T. Large anomalous Hall effect in a noncollinear antiferromagnet at room temperature. Nature 527, 212-215 (2015).

13. Nayak, A. K. et al. Large anomalous Hall effect driven by a nonvanishing Berry curvature in the noncolinear antiferromagnet $\mathrm{Mn}_{3} \mathrm{Ge}$. Sci. Adv. 2, e1501870 (2016).

14. Kuroda, K. et al. Evidence for magnetic Weyl fermions in a correlated metal. Nat. Mater. 16, 1090-1095 (2017). 
15. Kida, T. et al. The giant anomalous Hall effect in the ferromagnet $\mathrm{Fe}_{3} \mathrm{Sn}_{2}-\mathrm{a}$ frustrated kagome metal. J. Phys.: Condens. Matter 23, 112205 (2011).

16. Ye, L. et al. Massive dirac fermions in a ferromagnetic kagome metal. Nature 555, 638-642 (2018).

17. Hou, Z. et al. Observation of various and spontaneous magnetic skyrmionic bubbles at room temperature in a frustrated kagome magnet with uniaxial magnetic anisotropy. Adv. Mater. 29, 1701144 (2017).

18. Beal, A. R. in Intercalated Layered Materials Vol. 6 (ed. Lévy, F.) 251-305 (D. Reidel Publishing Company, Dordrecht, 1979).

19. Anzenhofer, K., van den Berg, J. M., Cossee, P. \& Helle, J. N. The crystal structure and magnetic susceptibilities of $\mathrm{MnNb}_{3} \mathrm{~S}_{6}, \mathrm{FeNb}_{3} \mathrm{~S}_{6}, \mathrm{CoNb}_{3} \mathrm{~S}_{6}$ and $\mathrm{NiNb}_{3} \mathrm{~S}_{6}$. J. Phys. Chem. Solids 31, 1057-1067 (1970).

20. Parkin, S. S. P., Marseglia, E. A. \& Brown, P. J. Magnetic structure of $\mathrm{Co}_{1 / 3} \mathrm{NbS}_{2}$ and $\mathrm{Co}_{1 / 3} \mathrm{TaS}_{2}$. J. Phys. C: Solid State Phys. 16, 2765-2778 (1983).

21. Friend, R. H., Beal, A. R. \& Yoffe, A. D. Electrical and magnetic properties of some first row transition metal intercalates of niobium disulphide. Philos. Mag. 35, 1269-1287 (1977).

22. Barišić, N. et al. High-pressure study of transport properties in $\mathrm{Co}_{0.33} \mathrm{NbS}_{2}$. Phys. Rev. B 84, 075157 (2011).

23. Beal, A. R. \& Liang, W. Y. Reflectivity spectra of some first row transition metal intercalates of $\mathrm{NbS}_{2}$. Philos. Mag. 33, 121-131 (1976).

24. Dheer, P. N. Galvanomagnetic effects in iron whiskers*. Phys. Rev. 156 637-644 (1967).

25. Checkelsky, J. G., Lee, M., Morosan, E., Cava, R. J. \& Ong, N. P. Anomalous Hall effect and magnetoresistance in the layered ferromagnet $\mathrm{Fe}_{1 / 4} \mathrm{TaS}_{2}$ : The inelastic regime. Phys. Rev. B 77, 014433 (2008).

26. Dijkstra, J., Zijlema, P. J., van Bruggen, C. F., Haas, C. \& de Groot, R. A. Bandstructure calculations of $\mathrm{Fe}_{1 / 3} \mathrm{TaS}_{2}$ and $\mathrm{Mn}_{1 / 3} \mathrm{TaS}_{2}$, and transport and magnetic properties of $\mathrm{Fe}_{0.28} \mathrm{TaS}_{2}$. J. Phys.: Condens. Matter 1, 6363-6379 (1989).

27. Machida, Y. et al. Unconventional anomalous Hall effect enhanced by a noncoplanar spin texture in the frustrated kondo lattice $\operatorname{Pr}_{2} \mathrm{Ir}_{2} \mathrm{O}_{7}$. Phys. Rev. Lett. 98, 057203 (2007).

28. Rößler, U. K., Leonov, A. A. \& Bogdanov, A. N. Chiral Skyrmionic matter in non-centrosymmetric magnets. J. Phys.: Conf. Ser. 303, 012105 (2011).

29. Bogdanov, A. N., Rößler, U. K., Wolf, M. \& Müller, K.-H. Magnetic structures and reorientation transitions in noncentrosymmetric uniaxial antiferromagnets. Phys. Rev. B 66, 214410 (2002).

30. Bogdanov, A. N. \& Yablonskiĭ, D. A. Contribution to the theory of inhomogeneous states of magnets in the region of magnetic-field-induced phase transitions. Mixed state of antiferromagnets. Sov. Phys. JETP 69, 142 (1989).

31. Sürgers, C., Fischer, G., Winkel, P. \& Löhneysen, H. v. Large topological Hall effect in the non-collinear phase of an antiferromagnet. Nat. Commun. 5, 3400 (2014).

32. Winker, R. Spin-Orbit Coupling Effects in Two-dimensional Electron and Hole Systems (Springer-Verlag, Berlin Heidelberg, 2003).

33. Silsbee, R. H. Spin-orbit induced coupling of charge current and spin polarization. J. Phys.: Condens. Matter 16, R179-R207 (2004).

34. Tsirkin, S. S., Souza, I. \& Vanderbilt, D. Composite Weyl nodes stabilized by screw symmetry with and without time-reversal invariance. Phys. Rev. B 96, 045102 (2017).

35. Blaha, P. et al. WIEN2K, An Augmented Plane Wave Plus Local Orbitals Program for Calculating Crystal Properties (Vienna University of Technology, Austria, 2001).

36. Sjöstedt, E., Nordström, L. \& Singh, D. J. An alternative way of linearizing the augmented plane-wave method. Solid State Commun. 114, 15-20 (2000).
37. Perdew, J. P., Burke, K. \& Ernzerhof, M. Generalized gradient approximation made simple. Phys. Rev. Lett. 77, 3865 (1996).

38. Singh, D. J. \& Nordström, L. Plane Waves, Pseudopotentials and LAPW Method (Springer, New York, 2006).

\section{Acknowledgements}

This work was supported by the U.S. Department of Energy, Office of Science, Basic Energy Sciences, Materials Science and Engineering Division. The authors thank insightful discussion with Daniel Phelan, I. Martin, F. Wu, Y. Xu, X. Dai, S. Chen and Z.-X. Shen. The EDS measurements were performed at the Center for Nanoscale materials in the Electron Microscopy Center, which was supported by the U.S. Department of Energy, Office of Science, Office of Basic Energy Sciences, under Contract No. DE-AC02-06CH11357. ChemMatCARS Sector 15 is principally supported by the Divisions of Chemistry (CHE) and Materials Research (DMR), National Science Foundation, under Grant NSF/CHE-1346572. Use of the Advanced Photon Source, an Office of Science User Facility operated for the US Department of Energy (DOE) Office of Science by Argonne National Laboratory, was supported by the US DOE under Contract DE-AC02-06CH11357. We acknowledge the computing resources provided on Blues, a high-performance computing cluster operated by Argonne's Laboratory Computing Resource Center.

\section{Author contributions}

N.J.G. and J.F.M. conceived the project. N.J.G. grew the crystals, performed the magnetic and magnetotransport measurements. J.S.J. contributed to the transport measurements. A.B. calculated the electronic band structure. Y.S.C. and J.Z. did the single crystal X-ray diffraction experiments. N.J.G. and J.F.M. wrote the manuscript. All authors contributed to the discussion of the results.

\section{Additional information}

Supplementary Information accompanies this paper at https://doi.org/10.1038/s41467 018-05756-7.

Competing interests: The authors declare no competing interests.

Reprints and permission information is available online at http://npg.nature.com/ reprintsandpermissions/

Publisher's note: Springer Nature remains neutral with regard to jurisdictional claims in published maps and institutional affiliations.

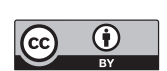

Open Access This article is licensed under a Creative Commons Attribution 4.0 International License, which permits use, sharing, adaptation, distribution and reproduction in any medium or format, as long as you give appropriate credit to the original author(s) and the source, provide a link to the Creative Commons license, and indicate if changes were made. The images or other third party material in this article are included in the article's Creative Commons license, unless indicated otherwise in a credit line to the material. If material is not included in the article's Creative Commons license and your intended use is not permitted by statutory regulation or exceeds the permitted use, you will need to obtain permission directly from the copyright holder. To view a copy of this license, visit http://creativecommons.org/ licenses/by/4.0/.

(c) The Author(s) 2018 